\title{
Langevin dynamics simulation of protein dynamics in nanopores at microsecond timescales
}

J. P. Mahalik, ${ }^{1}$ Jeffrey Cifello, ${ }^{1}$ and Murugappan Muthukumar ${ }^{1}$

Department of Polymer Science and Engineering, University of Massachusetts Amherst, Massachusetts ${ }^{\mathrm{a})}$

With rapid advancement in the fields of nanopore analysis of protein, it has become imperative to develop modeling framework for understanding the protein dynamics in nanopores. Such modeling framework should include the effects of electro-osmosis, as it plays significant role during protein translocation in confinement. Currently, the molecular dynamics simulations that include the hydrodynamic effects are limited to a timescale of few $100 \mathrm{~ns}$. These simulations give insight about important events like protein unfolding which occurs in this timescale. But many electrophoresis experiments are limited by a detector resolution of $\sim 2.5 \mu \mathrm{s}$. Analytical theory has been used to interpret protein dynamics at such large timescale. There is a need for molecular modeling of more complex environment and protein shapes which cannot be accounted for by analytical theory. We have developed a framework to study globular protein dynamics in nanopores by using langevin dynamics on a rigid body model of protein and the hydrodynamics is accounted by analytical theory for simple cylindrical nanopore geometry. This framework has been applied to study the dynamics of Ubiquitin translocation in $\mathrm{SiN}_{x}$ nanopore by Nir et $\mathrm{al}^{26}$. They have reported 7 times decrease in average dwell time of the protein inside the nanopore in response to a small change in $\mathrm{pH}$ from 7.0 to 7.2 and the modification of protein charge was attributed for such drastic change. Closer examination using our simulation revealed that the electro-osmotic effects originating due to very small change in the surface electrostatic potential of the nanopore could lead to such a drastic change in protein dynamics.

a)Electronic mail: jmahalik@umass.edu 


\section{INTRODUCTION}

Nanopore technology provides exciting opportunities for characterizing proteins, it probes them at a single molecule level, typically requires very small amount of sample $(\sim \mathrm{nM})$, and is a label free method ${ }^{1,2}$. This technology has been used in many applications, such as in protein sensing ${ }^{2,3}$, monitoring conformational changes of proteins such as folding/ unfolding ${ }^{4-8}$, and for self-assembly of proteins inside a nanopore ${ }^{9-11}$. Recently, there has been a lot of development and numerous opportunities in nanopore analysis of proteins, as evident from the large number of review articles in this particular field in just the past few years ${ }^{1-3,12-16}$. Protein analysis using nanopores is an indirect method wherein the structural and/or dynamic properties of a protein molecule are interpreted through ionic current trace. When an electric field is applied across a nanopore immersed in a hypertonic solution, a steady baseline open pore ionic current ( $\sim \mathrm{pA}$ to $\mathrm{nA})$ is observed. When a protein of size at least comparable to that of the nanopore diameter moves through it, total ionic flux is reduced, leading to a detectable reduction in the ionic current for certain duration of time. This current is referred as blockade current and it has two important characteristics: blockade current depth with respect to the open pore current, and the blockade duration (there could be multiple blockade depth levels). Many factors could influence the conformation and motion of a protein molecule inside the nanopore, such as interaction of protein molecule with the nanopore, confinement effect, and flow field of the aqueous media. Interpreting how protein structure and dynamics may be influenced by so many factors can be challenging based on just two sets of information: blockade current depth and blockade duration. A complimentary direct observation technique may facilitate such interpretation. All atomistic Molecular dynamics (MD) has been used to understand conformational changes of protein inside nanopores ${ }^{17-19}$, translocation dynamics ${ }^{20-22}$, protein sensing ${ }^{23}$, which typically happen in a timescale of less than or of the order of 100 ns. Coarse grain models have also been used to investigate protein translocation. Using Martini CG forcefield Haridasan et al ${ }^{24}$ investigated the translation of streptavidin though nanopores of different diameter, demonstrating how strong confinement can slow down protein dynamics due to strong protein-pore interactions. This simulation was of the timescale of few 100 ns. However, electrophoresis experiments are limited by their detector resolution at $\sim 2.5 \mu s^{25,26}$, the lowest dwell time reported for $\mathrm{RNase}^{25}$ and Ubiquitin ${ }^{26}$ is $\sim \mu s$. Besides the average dwell time, the dwell 
time distribution spans tens of $\mu s$, if not hundreds of $\mu s$. Analytical theory has been used to interpret the protein dynamics at such large timescales ${ }^{25,27}$. However, analytical calculations may not be applied to all situations, especially for more complex environment and protein shapes, where the details of the system such as shape of the protein can significantly impact the dynamics. A generalized framework is needed for probing protein dynamics in confinement at a timescale of $\mu s$ or higher. Ustach and Faller ${ }^{28}$ proposed raspberry model for protein-like particle dynamics in cylindrical confinement at large timescales. They calculated the hydrodynamic resistances of ellipsoid raspberries to translational and rotational motion using lattice Boltzmann and highlighted the need for considering more complex rigid shapes.

A protein molecule experiences different kinds of forces during its expedition through a nanopore. Unlike DNA or RNA, a protein molecule is typically made up of a mixture of positive and negative charged residues. The externally applied electric field exerts electrophoretic force on positively charged residues in one direction and pushes the negatively ones in the opposite direction, resulting in a net translational and rotational motion of the protein in a particular direction. Secondly, depending on the nature of charge of the nanopore and the polarity of the applied voltage, electroosmotic driving force may become significant, leading to either enhancement or reduction of the protein velocity. Also, the interaction of the protein with the nanopore walls and the confinement effects of the nanopore may influence the motion of the protein molecule. In addition to influencing the dynamics of the protein, the confinement effect of the nanopore may cause structural changes in the protein molecule such as unfolding ${ }^{17-19}$. We will consider only the simplest case when the protein moves through the nanopore without any significant conformational changes ${ }^{2}$. Muthukumar ${ }^{27}$ modeled the effects of counterion cloud, external electric field, confinement effect inside nanopore and protein-pore interaction on the dynamics of protein translocation through nanopore using analytical theory. Without adequately accounting for all this factors, the interpretation from the single molecule electrophoresis experiments can be misleading. For example, at high salt the effective charge on a protein molecule $(Q)$ could be reduced by about an order of magnitude and the diffusivity of the protein $(D)$ could be reduced significantly due to the confinement effects and the protein-pore attraction. Simply using the Einstein equation $\left(\mu=Q D / k_{B} T\right)$ for interpreting the protein dynamics can be erroneous. Where $\mu$ is the electrophoretic mobility of the protein, $k_{B} T$ is the Boltzmann 
constant times the absolute temperature, $Q$ is typically taken to be overall charge of the protein without accounting for its counterion cloud, and $D$ is generally approximated from Stokes-Einstein relation for a sphere in a free medium. This analytical approach provides a good starting point for estimating the effects of some of the factors on the dynamics of protein during translocation through a nanopore. Specific details can become important in many situations, for example, Liu et $\mathrm{al}^{29}$ investigated the effect of wettability condition inside the nanopore on the CG protein translocation velocity, stronger attraction between the water molecules and the nanopore can slow down the protein dynamics significantly in hydrophilic nanopores instead of protein-nanopore interactions. Such insight could be obtained by molecular modeling, otherwise it could have been wrongly attributed to some other factor.

Nir et $\mathrm{al}^{26}$ investigated translocation of Ubiquitin through a $\mathrm{SiN}_{x}$ nanopore of diameter comparable to its size (3.6 nm diameter nanopore). They observed that the average protein dwell time decreased by about a factor of 7 , with a small change in $\mathrm{pH}$ from 7.0 to 7.2 from $18.4 \mu \mathrm{s}$ to $2.6 \mu \mathrm{s}$, respectively. For $\mathrm{pH}$ values higher than 7.2 , the translocation events were too fast to be detected. For $\mathrm{pH}$ values lower than 7.0, the capture rate dramatically dropped, making it difficult for statistical analysis. Such a drastic response of translocation time to such a small change in $\mathrm{pH}$ was attributed to the modification of the protein charge in response to change in $\mathrm{pH}$. However, analysis of the Ubiquitin sequence ${ }^{30}$ revealed that it has only one Histidine residue and that is also expected to be in deprotonated state for $\mathrm{pH}$ range 7.0-7.2 $2^{31}$. Therefore, the electrostatic effects must be originating from the nanopore. At a high salt concentration of $1.5 \mathrm{M} \mathrm{KCl}$, we expect that the electrostatic interactions will be highly screened. Most likely the drastic change in protein dynamics is originating from electroosmotic interactions. In order to account for hydrodynamics in such systems, generally explicit solvent molecules are considered atomistically ${ }^{20-22}$ or $\mathrm{CG}^{24,29}$, or less often through hybrid model: coupling MD of the biomolecule with hydrodynamic interactions from Lattice-Boltzmann (LB) calculations ${ }^{32}$. Such hybrid model can still be computationally expensive, requiring numerical LB calculations over the grids at different timesteps. For simplified geometries such as a cylindrical nanopores of sufficiently large diameter, continuum approximations can be used to obtain fluid velocity profile as a function of nanopore surface potential, applied electric field, fluid viscosity, nanopore diameter and salt concentration ${ }^{33}$. At sufficiently low Reynolds number, we can assume that the biomolecule experiences the 
electro-osmosis force but it does not influences the fluid flow profile around it. Such simplified assumption leads to only one calculation of fluid flow profile in the beginning of MD calculations, the fluid velocity profile can then be related to particle dynamics through langevin dynamics. Another computational challenge is accounting for different kinds of interactions (bonds, angular, dihedral, etc) between the residues during translocation. If the protein unit is small enough and globular to slide through the nanopore and the external forces are weak enough such that the internal fluctuations within the protein structure can be neglected, then we can model the protein unit as a rigid body of any arbitrary shape (depending on the Protein Data Bank (PDB) structure) instead of restricting to symmetric shapes $^{28}$. The residues can interact with the nanopore through short-range and long-range pairwise interactions based on the nature of the residues.

We used langevin dynamics simulations to study the dynamics of electrophoretically driven Ubiquitin through a $\operatorname{SiN}_{x}$ nanopore of diameter $3.6 \mathrm{~nm}^{26}$. The electro-osmotic effects were accounted for analytically and the protein is modeled as a rigid body. With these assumptions, we were able to monitor Ubiquitin dynamics inside the nanopore upto tens of $\mu s$ and we demonstrate that the electro-osmosis can drastically reduce the dwell time of the Ubiquitin. A surface potential of $\sim m V$ can have a significant impact on the dynamics, changing the qualitative dynamics of Ubiquitin from diffusion like to drift like.

\section{MODEL AND SIMULATION DETAILS}

Simulations were performed at two different length/time scales: all atomistic molecular dynamics (MD) simulations and coarse grained langevin dynamics (LD) simulations. All atomistic MD simulation was performed on a Ubiquitin molecule immersed in bulk $1.5 \mathrm{M}$ $\mathrm{KCl}$ aqueous solution in nanosecond timescale. The stability of the protein structure was determined in the bulk without and with externally applied electric field. The root mean square deviation (RMSD) of the protein backbone as a function of time was used to gauge the stability of the protein in the timescale of nanoseconds. MD simulations were performed with CHARMM protein potential and TIP3P water model using NAMD ${ }^{34}$ software. Since the protein was found to be stable in the bulk (both without and with the externally applied electric field), the next stage of the simulation was conducted at a timescale of microseconds on a coarse grained $(\mathrm{CG})$ model of the protein, assuming a rigid protein structure. The 
coarse graining was done at the residue level, each CG bead represents one residue. A rigid protein structure implies that the relative position of every residue with respect to each other is kept fixed during the motion of the protein unit. Such a simplification allows turning off of interaction among beads within the protein, their dynamics is determined through their interaction with the environment. The second part of the simulation is described in detail below:

Langevin dynamics simulation was used to investigate the Ubiquitin dynamics in the nanopore, using LAMMPS ${ }^{35}$. The Langevin equation for the $j^{\text {th }}$ component of the position vector of the $i^{\text {th }}$ bead $\left(r_{i j}\right)$ is given by,

$$
m_{i} \frac{d^{2} r_{i j}}{d t^{2}}=-\frac{m_{i}}{\tau_{d a m p}}\left(\frac{d r_{i j}}{d t}\right)+f_{i j}-\nabla_{j} U_{i}+\frac{m_{i}}{\tau_{d a m p}} v_{f j}+q_{i} E_{j}
$$

where $m_{i}$ is the mass, $\tau_{d a m p}$ is the damping factor, and $v_{f j}$ is the $j^{\text {th }}$ component of the local fluid velocity acting on the $i^{\text {th }}$ bead. $f_{i j}$ is the $j^{\text {th }}$ component of the random force acting on the $i^{\text {th }}$ bead obeying the fluctuation-dissipation theorem with its magnitude proportional to $\sqrt{k_{B} T m_{i} /\left(d t \tau_{d a m p}\right)}\left(k_{B} T\right.$ being the Boltzmann constant times the absolute temperature and $d t$ is the timestep), its magnitude and direction being randomized using random numbers. $m_{i}$ is taken to be same for all the beads and Stokes' law was used to estimate the $\tau_{d a m p}$, $m_{i} / \tau_{\text {damp }}=3 \pi \eta \sigma$, where $\eta$ is the viscosity of the water at $298 \mathrm{~K}$ equal to $1.0 \times 10^{-3}$ poise. $\tau_{\text {damp }}$ is estimated to be 51 femtoseconds, based on these parameters. Refer Table I for $m_{i}$ and $\sigma$ (diameter of a CG residue) values. The second last term on the RHS of Eq. 1 is the electro-osmotic force due to fluid velocity $v_{f j}$ and the last term is the electrophoretic force experienced by a bead possessing charge $q_{i}$ due to external electric field $E_{j}$.

$U_{i}$ is the net potential on the $i^{\text {th }}$ bead due to contributions from the repulsive excluded volume interactions and the screened Coulombic interactions. The excluded volume interaction is modeled as Lennard Jones (LJ) potential

$$
\begin{array}{r}
U_{L J}=4 \epsilon\left[\left(\frac{\sigma}{r}\right)^{12}-\left(\frac{\sigma}{r}\right)^{6}\right]-4 \epsilon\left[\left(\frac{\sigma}{r_{c}}\right)^{12}-\left(\frac{\sigma}{r_{c}}\right)^{6}\right], r \leq r_{c} \\
=0, r>r_{c}
\end{array}
$$

where $\sigma$ and $\epsilon$ are parameters. We have chosen the cut-off $r_{c}$ to be $1.12 \sigma$ to model excluded volume potential. The pairwise electrostatic interaction potential between the $i^{\text {th }}$ bead possessing a charge of $q_{i}$ and the $j^{\text {th }}$ bead with a charge of $q_{j}$ separated by a distance $r$ is 
given by the screened Coulombic interaction

$$
U_{C}=\frac{q_{i} q_{j}}{4 \pi \epsilon_{0} \epsilon_{r} r} \exp (-\kappa r)
$$

where $\epsilon_{0}$ is the permittivity of the vacuum, $\epsilon_{r}$ is the dielectric constant of the medium taken to be 60 based on $1.5 \mathrm{M} \mathrm{KCl}$ aqueous solution ${ }^{36}$. The inverse Debye length $\kappa=$ $\sqrt{8 \pi \lambda_{B} N_{A} \times 10^{3} I}=0.454 \AA^{-1}$, where $I$ is the ionic strength in molar, $N_{A}$ is the Avogadro number and $\lambda_{B}$ is the Bjerrum length given as, $\lambda_{B}=e^{2} /\left(4 \pi \epsilon_{0} \epsilon_{r} k_{B} T\right)$, e being the electronic charge.

The velocities and positions of the protein beads are updated by the rigid body algorithm adopted from Miller et $a l^{37}$ in $\mathrm{LAMMPS}^{35,38}$. The position of the beads belonging to the nanopore are kept fixed. All variables in our simulations are expressed in dimensionless LJ unit, fully consistent with LAMMPS.

\section{A. Coarse-graining scheme}

\section{Protein}

CG model was built from all atomistic model reported in the PDB (ID: 1UBQ) ${ }^{30}$. The C- $\alpha$ position for each residue was taken as the CG residue center and every residue was assigned a diameter of $3.8 \AA^{39}$. ASP and GLU were assigned -0.2e charge and LYS and ARG were assigned +0.2 e charge. The effective charge fraction on the protein is about 0.2 due to the counterion cloud at high salt ${ }^{27}$. At around $\mathrm{pH}=7$, the histidine is neutral ${ }^{31}$, hence it is assigned zero charge.

\section{Nanopore}

The nanopore parameters are adopted from the experiment ${ }^{26}$. Nanopore was made up of $3.8 \AA$ diameter $\mathrm{CG}$ beads such that the distance from the center of the pore axis to the bead is $1.99 \mathrm{~nm}$ (Pore radius + radius of the CG bead), resulting in an available pore diameter of $3.6 \mathrm{~nm}$ for translocation. The length of the pore was originally taken to be $8 \mathrm{~nm}$, but the number of capture events were extremely low for statistical analysis, for few cases. We redefined the problem such that we don't have to worry about the low capture events. We modeled a nanopore of diameter $3.6 \mathrm{~nm}$ and a length of $80 \mathrm{~nm}$ (10 times longer than the original nanopore) and started with an initial condition of protein being placed at the center of the nanopore. The dynamics of the protein was then monitored to evaluate the effect of 
TABLE I: Fundamental parameters in the simulation from real to LJ units

\begin{tabular}{ccc} 
Parameter & Real Units & LJ units \\
\hline Mass $\left(m_{0}\right)$ & $110 \mathrm{~g} / \mathrm{mol}$ (Average mol wt of residue) & 1 \\
Length $(\sigma)$ & $3.8 \AA$ (Diameter of CG residue) & 1 \\
Time $(\tau)$ & $2.53 \mathrm{ps}$ & 1 \\
Energy $(\epsilon)$ & $1 k_{B} T$ at $298 \mathrm{~K}$ & 1 \\
Force $(F)$ & $10.82 \mathrm{pN}$ & 1 \\
Charge $(q)$ & 1 electronic charge & 12.14 \\
Electric field $(E)$ & $1 \mathrm{~V} / \AA$ & 0.0821
\end{tabular}

various factors only on the translocation events and not the capture events.

\section{Characterization}

The results were characterized in three different ways: (a) First Passage Time $(\tau)$ : First time the protein's center of mass crosses a particular distance starting from an original position at the center of the axis of the pore. (b) z-Mean Square Displacement (MSD): Squared displacement of center of mass from its original position along the nanopore axis. (c) Angular orientation: The angle between the principal axes of Ubiquitin (vector from $76^{\text {th }}$ residue to $20^{\text {th }}$ residue of $\mathrm{PDB}$ ) and the nanopore axis. 600 independent simulations were conducted and histogram analysis was done to obtain mean first passage time $\langle\tau\rangle$, and ensemble averaging was done for the other two parameters.

\section{RESULTS AND DISCUSSION}

\section{A. All Atomistic Simulation}

The RMSD of the protein backbone was determined as a function of time for two cases: (a) without electric field (b) with applied electric field of $125 \mathrm{mV} / 8 \mathrm{~nm}$ applied in any particular orthogonal direction. The Ubiquitin has a radius of gyration $\left(R_{g}\right)$ of about $1.2 \mathrm{~nm}$ and the RMSD was determined to be within $2 \AA$ ( $<10 \%$ of the diameter of the protein) for all the cases (observation time $=2 \mathrm{~ns}$ ) (Fig. 1). Since the protein retained its structural integrity as expected for a globular protein, therefore the protein can be approximated as a rigid unit for 
coarse graining purpose. We are assuming that on an average the instantaneous fluctuations in the conformations of the protein can be neglected for the large time scale simulations. Such approximations may not be appropriate for intrinsic disordered proteins, where the RMSD of the backbone is expected to fluctuate a lot. As the $R_{g}(=1.2 \mathrm{~nm})$ of the protein is smaller than the radius of the nanopore $(=1.8 \mathrm{~nm})$, therefore the protein need not deform in order to move inside the nanopore. The protein is elongated (maximum distance between residues is $3.7 \mathrm{~nm}$ ), hence it cannot enter the nanopore in any orientations. Later in CG simulations, we start with the Ubiquitin principal axis either pointing towards the positive z-direction or the negative z-direction and let it freely evolve into some orientation by keeping it's center of mass fixed at the initial position, before starting the simulation. In CG simulations we also investigated the effect of electro-osmosis on the dynamics of Ubiquitin. One may ask, what is the impact of the shear on the conformation of the Ubiquitin? At our operational shear rate, does Ubiquitin deform or unravel? According to the all atomistic simulation studies by Walinda et $\mathrm{al}^{40}$ on Ubiquitin, upto a shear rate of $1.4 \times 10^{9} \mathrm{~s}^{-1}$, the protein do not show internal structural fluctuations in $20 \mathrm{~ns}$ simulation time. In our study, the maximum shear rate is two orders of magnitude smaller, hence our assumption of rigid body model for Ubiquitin seems reasonable in these conditions. Moreover, there is strong experimental evidence that small proteins can travel through $\mathrm{SiN}_{x}$ nanopore in folded conformation ${ }^{41}$.

\section{B. Effect of $\mathrm{pH}$ on the Protein Charge}

The protein is made up of 76 residues, out of which 11 are positive and 11 are negative and one Histidine. Since the pH of the medium is between 7.0-7.2, therefore the charge of Histidine is expected to be zero ${ }^{31}$. The net charge of the protein is zero and for the given experimental conditions $(\mathrm{pH}=7.0$ and $\mathrm{pH}=7.2$ ), the charge of the protein is not expected to change. Therefore, the observed dramatic change in translocation time due to small change in $\mathrm{pH}$ cannot be attributed to modification in the protein charge ${ }^{26}$.

\section{Effect of $\mathrm{pH}$ on the Nanopore Surface}

In order to determine the effect of $\mathrm{pH}$ on the surface charge of $\mathrm{SiN}_{x}$, experimental literature, Ref. ${ }^{42}$ was followed. According to Ref. ${ }^{42}$, the $\mathrm{SiN}_{x}$ surface potential/surface charge 


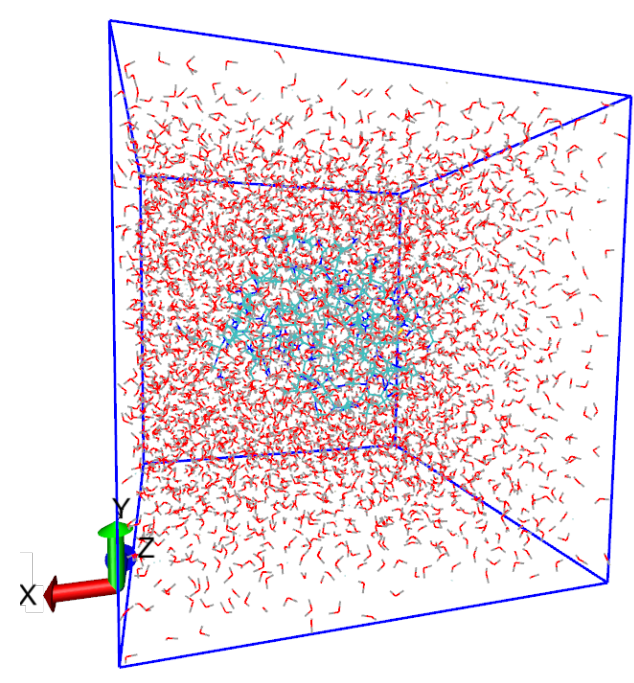

(a)

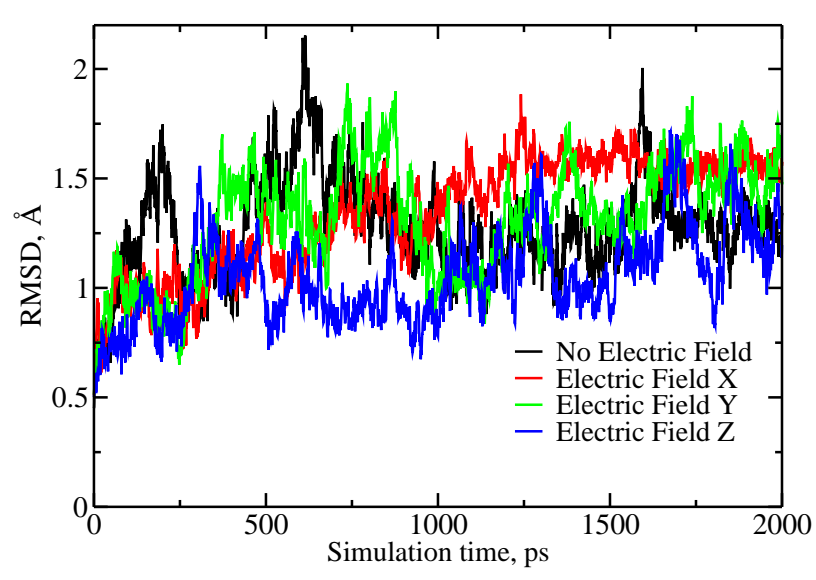

(b)

FIG. 1: All atomistic MD simulation results demonstrating the stability of Ubiquitin molecular structure in bulk saline solution, irrespective of the presence of an external electric field $(|E|=0.0015625 \mathrm{~V} / \AA)$. (a) Snapshot of a Ubiquitin molecule immersed in 1.5

$\mathrm{M} \mathrm{KCl}$ aqueous solution. The blue stick model in the center of the simulation box is a Ubiquitin molecule which is surrounded by water and dissociated $\mathrm{KCl}$ ions. (b) RMSD of the protein backbone as a function of time shows that the fluctuations are within $2 \stackrel{\circ}{\text { for }}$ all the cases which is less than $10 \%$ of the diameter of the protein $\left(=2 \mathrm{x} R_{g}=24 \AA\right.$ ), in a simulation time of 2000 ps. Different cases are: no electric field and constant electric field in any orthogonal direction.

are $3.6 \mathrm{mV} / 0.14 \mu \mathrm{Ccm}^{-2}$ and $-7.2 \mathrm{mV} /-0.11 \mu \mathrm{Ccm}^{-2}$ corresponding to $\mathrm{pH}$ values of 7.0 and 7.2 , respectively, at $0.01 \mathrm{M} \mathrm{NaCl}$. At $1.5 \mathrm{M} \mathrm{KCl}$, we expect the magnitude of the surface potential and surface charge to be reduced, based on their trend for two different salt concentrations: $0.001 \mathrm{M}$ and $0.01 \mathrm{M} \mathrm{NaCl}$. Is it possible to observe such a dramatic change in translocation time distribution with such small values of surface potential and surface charge? Since, we do not know the exact values of these parameters, hence we systematically vary the surface potential between $5 \mathrm{mV}$ to $-5 \mathrm{mV}$.

When the simulation was conducted with the original nanopore length $(8 \mathrm{~nm})$, by initiating Ubiquitin near the nanopore mouth, the number of capture events was very low for certain cases. Since Ubiquitin consists of equal number of positive and negative residues, 


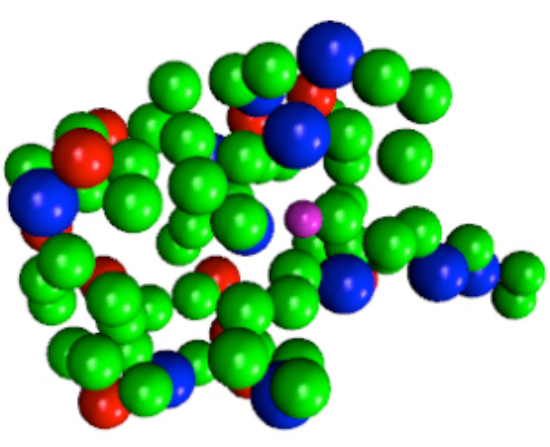

(a)

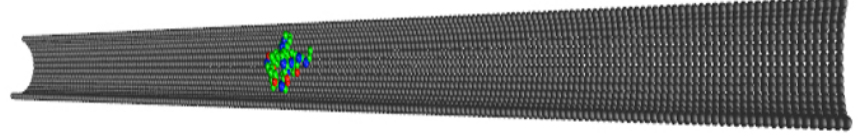

(b)

FIG. 2: Coarse grain (CG) model of Ubiquitin and the nanopore. (a) Residue based CG model of Ubiquitin derived from it's all atomistic coordinates (PDB ID: $1 \mathrm{UBQ}^{30}$ ), protein is modeled as a rigid-body. Different residues are color coded based on their charge, neutral: green; positive: blue; negative: red, the lone histidine residue is represented in magenta color. In total there are 76 residues, including 11 positive residues and 11 negative residues, Histidine being neutral at $\mathrm{pH}=7.0 \pm 0.2^{31}$. (b)Initial configuration of Ubiquitin in the simulation box, centered in a $\mathrm{CG} \mathrm{SiN}_{x}$ nanopore of $3.6 \mathrm{~nm}$ diameter and $80 \mathrm{~nm}$ length. The model nanopore diameter was same as the experiment but it's length was ten times larger ${ }^{26}$. Such a model setup allows understanding of the role of different driving forces on the dynamics of translocation of Ubiquitin by overcoming extremely low capture rate corresponding to certain experimental $\mathrm{pH}$ conditions.

therefore driving it into the nanopore with just electrophoretic force can be challenging. Unless driven by electro-osmosis, the total number of successful capture events were extremely low for further statistical analysis. Since the goal of our study was to study the effects of electrostatic parameters on the dynamics of translocation (not capture), we conducted our simulation in a very long nanopore $(80 \mathrm{~nm})$, with the Ubiquitin initiated at the center of the nanopore. The dynamics of the Ubiquitin translocation was investigated for two different sets of forces: (a) protein-pore electrostatic interactions (b) electro-osmosis effects from the charged nanopore. 


\section{Protein-pore Electrostatic Interactions}

We only consider electrostatic interactions, but the non-specific interactions between the protein molecule and the nanopore are omnipresent. We assume that the small change in pH from 7.0-7.2, modulates only the electrostatic environment inside the nanopore. We considered the simplified case of short-range excluded volume interaction between the residues and nanopore beads and how the additional electrostatic interactions impact the Ubiquitin dynamics. If there is any impact at such simplified conditions then its worth considering more complex short-range interactions. We determined the charge on individual nanopore CG beads $\left(q_{n}\right)$ from Ref. ${ }^{42}$ to be about -0.001 e at $7.2 \mathrm{pH}$. We investigated how the screened coulombic interactions between the charged residues and nanopore affects the first passage time of Ubiquitin COM along the nanopore axis for three different $q_{n}=0,-0.001 \mathrm{e}$, and 0.01e. Since the Debye length $(2.2 \AA)$ is smaller than the CG diameter of a residue (3.8 $\AA$ ), hence we do not expect any impact of electrostatic interactions. As shown in Fig. 3(a), we observed that the histograms for the three $q_{n}$ values are very similar. In fact for all the $z$ values along the nanopore, the histograms were quite similar, which is evident from the plot of mean first passage time $(\tau)$ versus $z$ (Fig. 3(b)). With increase in $z, \tau$ increased linearly and symmetrically across the nanopore plane, and $\tau$ corresponding to $z=8 \mathrm{~nm}$ is about $4 \mu \mathrm{s}$. This demonstrates that the electrostatic interaction is not responsible for such a drastic change in the average translocation time in response to a small change in $\mathrm{pH}$ in the experiment. Next, we investigated the role of electro-osmosis on the mean first passage time.

\section{Electro-osmosis Effects}

Using the analytical expression derived in Ref. ${ }^{33}$, the fluid velocity profile inside $\left(v_{f}\right)$ the nanopore is obtained as shown in Eq. 5 , where $\phi_{0}$ is the surface potential, $E$ is the applied electric field, $\epsilon$ is the dielectric of the medium, $\eta$ is the viscosity of the medium, $I_{0}$ is the zeroth order Bessel function of the first kind, $r$ is the radial distance from the pore axis and $a$ is the radius of the nanopore. Since the pore is very long, therefore the velocity profile is assumed to be independent of the position of the axis (fully developed flow assumed throughout the length of the nanopore), and is only a function of the radial 


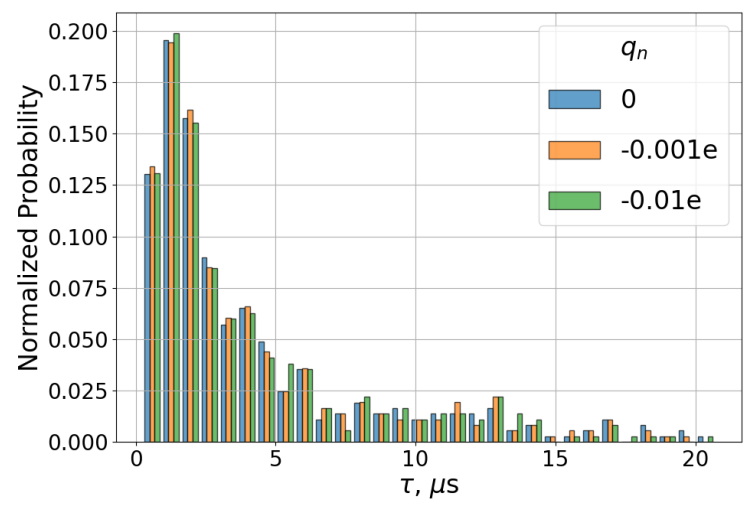

(a)

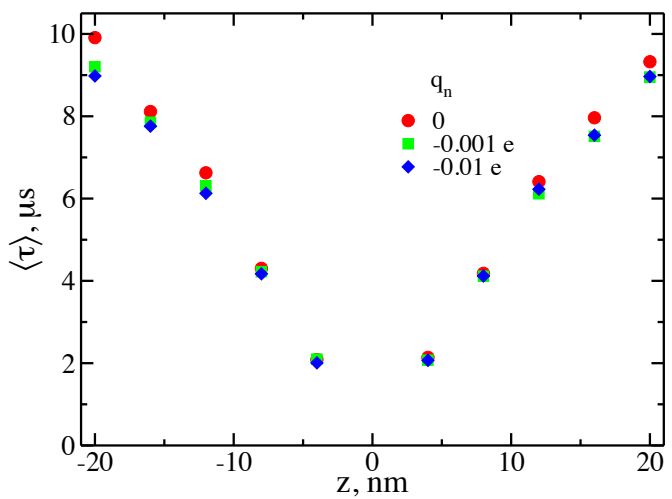

(b)

FIG. 3: Effects of electrostatic interaction between protein-nanopore on the dynamics of electrophoretically driven Ubiquitin translocation. The charge per CG nanopore bead $\left(q_{n}\right)$ is -0.001 e at $\mathrm{pH}=7.2$, we also studied the effect at higher surface charge. (a) A histogram for the first passage time of Ubiquitin at $+8 \mathrm{~nm}$, initiated at the center of the nanopore, shows insignificant effect of $q_{n}$. Other histograms at $+4 \mathrm{~nm}$ intervals also showed qualitatively similar behavior. (b) The mean first passage time obtained at regular intervals along the nanopore axis is almost symmetric across the nanopore central plane for all the cases and is insensitive to the charge for any given $z$ of the nanopore at such high salt concentration of $1.5 \mathrm{M} \mathrm{KCl}$, as expected.

distance from the nanopore axis. The fluid velocity obtained for the $i^{\text {th }}$ bead located at $r$ along the nanopore axis is multiplied by a factor of $m_{i} / \tau_{d a m p}=3.58 \times 10^{-12} \mathrm{~kg} / \mathrm{s}$ to convert it into electro-osmotic force experienced by the residue. This force is then converted to LJ units (Table I) and polynomial fitted (11 ${ }^{\text {th }}$ order), the polynomial expression is used as an input parameter for the langevin equation (Eq. 1).

$$
v_{f}(r)=-\frac{\phi_{0} E \epsilon}{\eta}\left[1-\frac{I_{0}(\kappa r)}{I_{0}(\kappa a)}\right]
$$

We assumed that the velocity profile of the fluid is not influenced by the presence of Ubiquitin. The fluid velocity $v_{f}$ is plotted as a function of $r$ for different $\phi_{0}$ as shown in Fig. 4, which is parabolic and its direction is influenced by the magnitude of $\phi_{0}$. For positive values of $\phi_{0}, v_{f}$ is in the negative z-direction, that explains the low capture rate reported in Ref. $^{26}$ at lower $\mathrm{pH}$. At lower $\mathrm{pH}$, the $\mathrm{SiN}_{x}$ nanopore is expected be more positive ${ }^{42}$. One may ask the applicability of the continuum approximation in such a narrow nanopore. 
According to Ref. ${ }^{43}$, the ratio of the nanochannel height to approximately the diameter of molecules filling the nanochannel determines whether or not continuum approximations is applicable. By comparing all atomistic molecular dynamics simulations results with the analytical calculations from Navier-Stokes equation, they demonstrated that if this ratio is above 100 then the continuum approximation is valid, below 10 it is not valid, in between the fit between theory and simulation results are not great. Since we are using a nanopore of diameter $3.6 \mathrm{~nm}$, considering diameter of water molecule of $0.27 \mathrm{~nm}$, this ratio is about 13.3. Hence, for our case the continuum approximation is not great, yet not invalid. It should be noted that our velocity is three orders of magnitude smaller than their system, hence the non-linear effects occurring at higher velocities may not be significant in our case. The continuum approximation may be alright for this system, it needs to be tested though.

Next, we investigated the effect of electro-osmosis on the dynamics of Ubiquitin. As shown in Fig. 5(a), there is a drastic effect of electro-osmosis on the first passage time histogram of Ubiquitin at $+8 \mathrm{~nm}$ along the nanopore axis. The first passage time which spanned till $20 \mu \mathrm{s}$ for $\phi_{0}=0 \mathrm{mV}$, spans till $1 \mu \mathrm{s}$ for $\phi_{0}=-3 \mathrm{mV}$. The histogram was symmetric on the other side of the nanopore corresponding to positive values of $\phi_{0}$ (not shown). Similar qualitative behavior was observed all along the nanopore as evident from the $\tau$ versus $z$ in Fig. 5(b). $\tau$ for positive $\phi_{0}$ are not plotted in the positive z-direction, and vice versa, due to insufficient statistics. $\tau$ versus $z$ shows linear behavior for all the cases of $\phi_{0}$. Although we cannot make a direct comparison with the experimental results reported in Ref. ${ }^{26}$ but the $\langle\tau\rangle$ values corresponding to $z=+8 \mathrm{~nm}$ are of the the same order of magnitude $(\sim \mu s)$ as the dwell time reported in Ref. ${ }^{26}$ for a $8 \mathrm{~nm}$ long nanopore, and it decreases with increase in the $\phi_{0}$ value. We do not have enough data to do statistical analysis for positive $\phi_{0}$ values corresponding to $z=8 \mathrm{~nm}$, but we expect $\langle\tau\rangle$ to increase further with increase in $\phi_{0}$ for longer simulations on a larger sample.

We also investigated the effect of $\phi_{0}$ on the z-component of the mean square displacement (z-MSD) as well as the orientation of the protein with respect to the nanopore axis. For the case of zero surface potential, the protein prefers to stay within the nanopore, as evident from a fluctuating z-MSD profile near $100 \mathrm{~nm}^{2}$ as shown in Fig. 6(a). For the other cases, z-MSD showed qualitatively different behavior, it increased as a function of time. The slopes of z-MSD increased as the magnitude of $\phi_{0}$ increased. The z-MSD for the positive $\phi_{0}$ values showed very similar trajectories as the corresponding negative $\phi_{0}$ (not shown). Such 


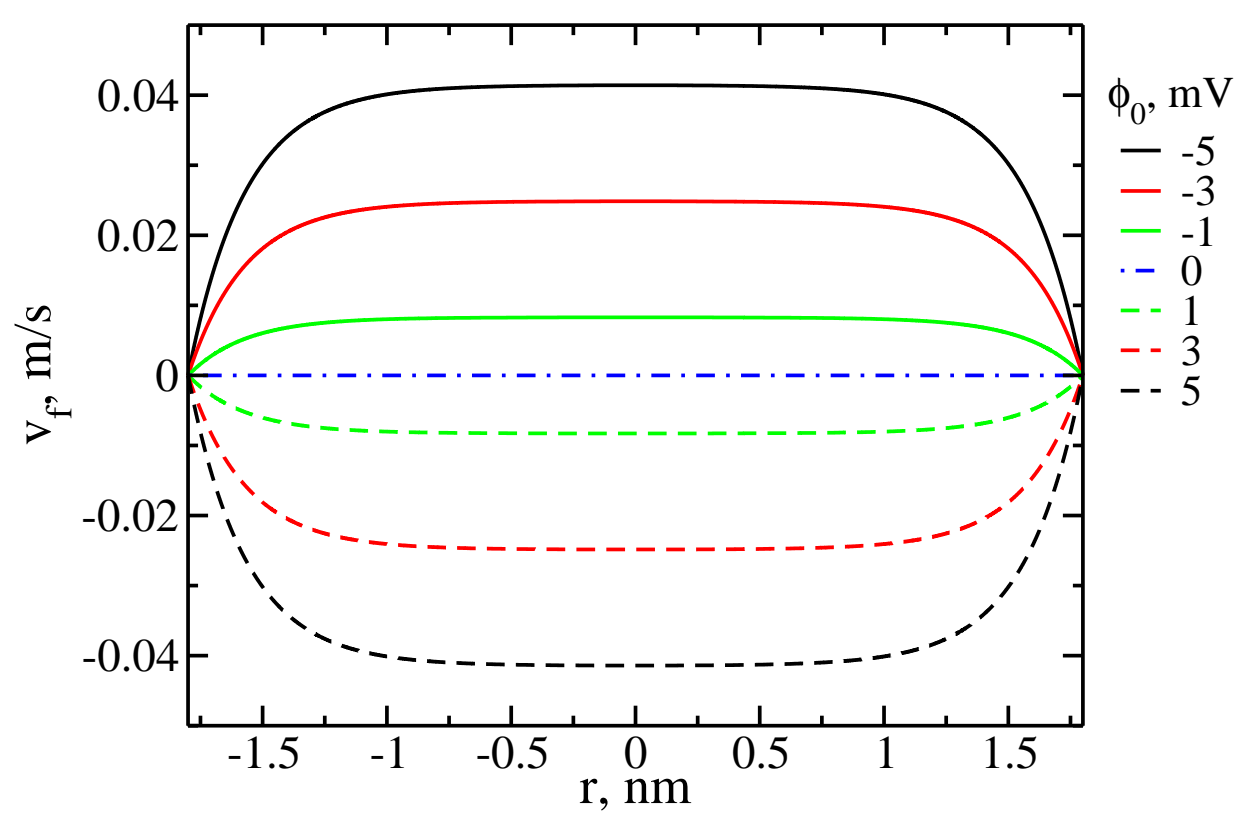

FIG. 4: $v_{f}$ as a function of $r$ is obtained using Eq. 5 for different nanopore surface potentials. The velocity is multiplied by a factor $m_{i} / \tau_{d a m p}$ (in real units) and then converted to LJ force units (Table. I) followed by fitting with a sixth order polynomial function. The polynomial function is used as an input parameter to model electrophoretic force in the langevin equation (Eq. 1).

qualitative trend in z-MSD is expected, based on their first passage time results. Besides translational dynamics, we also investigated the rotational dynamics of Ubiquitin while undergoing translocation through the nanopore. As shown in Fig. 6(b), the orientation of the principal axes (vector from $76^{\text {th }}$ residue to $20^{\text {th }}$ residue) is independent of $\phi_{0}$ and is dependent only on it's original orientation at initial time. Although Ubiquitin wiggles a lot (as evident from the thick average value of the orientation) but it cannot flip to the opposite direction due to it's elongated shape. On an average it prefers an angle of about $34^{\circ}$ or $150^{\circ}$ with the nanopore axis depending on it's initial orientation, probably due to the distribution of charged residues along the protein. We did not observe any effect of the initial orientation on it's translational dynamics. 


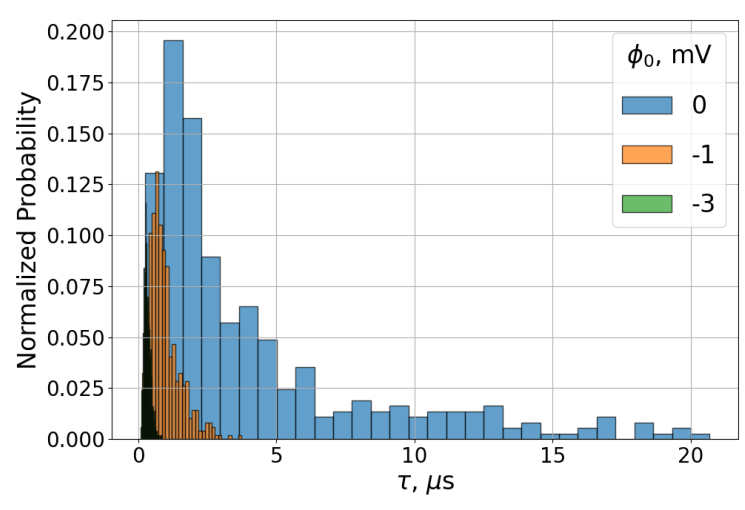

(a)

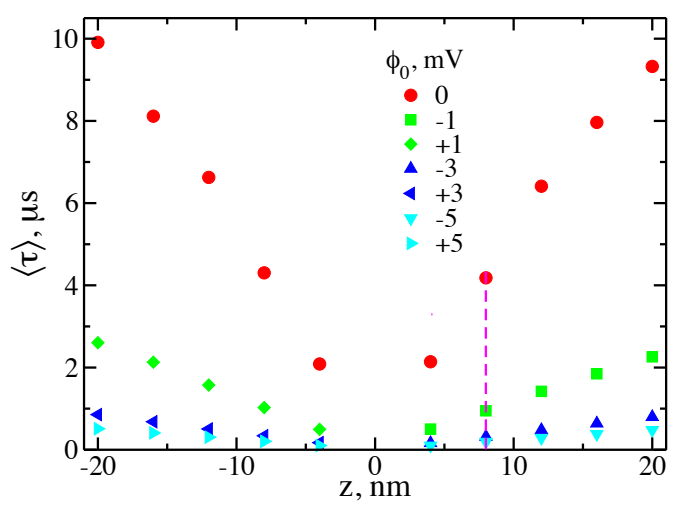

(b)

FIG. 5: Effect of electro-osmosis on the dynamics of electrophoretically driven Ubiquitin.

(a) Histograms for the first passage time of Ubiquitin at $+8 \mathrm{~nm}$ for three different nanopore surface potentials showing significant decrease in first passage time with decrease

in surface potential. For positive surface potentials, there were insignificant number of events corresponding to positive $\mathrm{z}$ values, hence not presented. Other histograms at an interval of $4 \mathrm{~nm}$ along the nanopore axis are qualitatively similar. (b) The mean first passage time $(\tau)$ obtained at regular intervals along the pore axis is significantly affected by the surface potential $\left(\phi_{0}\right)$; higher the magnitude of $\phi_{0}$, lower the $\tau$. Negative surface potential biases the protein motion in the positive $z$-direction, and vice-versa.

\section{CONCLUSIONS}

We have developed a framework for determining the large timescale $(\sim 10 \mu s)$ dynamics of globular proteins in nanopores. The globular protein can be assumed to be a rigid body, if the protein is smaller along two of its principal axis than the nanopore diameter (so that it fits without getting deformed) and the external force is weak enough such that the conformational fluctuations of the protein can be neglected (high shear can unravel the protein). For cylindrical nanopores of sufficiently large diameter, the electro-osmotic effects can be accounted for by analytical expressions from continuum model. Use of analytical expression for modeling the fluid velocity simplifies the calculations significantly, making it feasible to do simulations of the order of tens of $\mu s$. With such framework, many different globular proteins can be studied. Investigation can be done on the role of charge distribution and overall charge of the protein on its dynamics. Moreover, the short-range interactions between residue-nanopore pairs can be easily included, if these parameters are known. Lastly, 


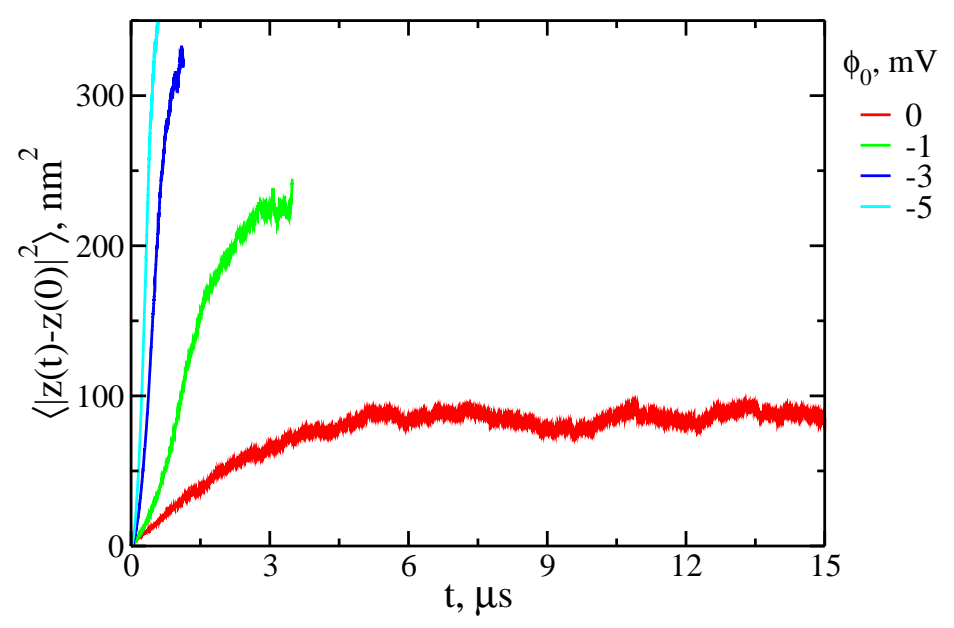

(a)

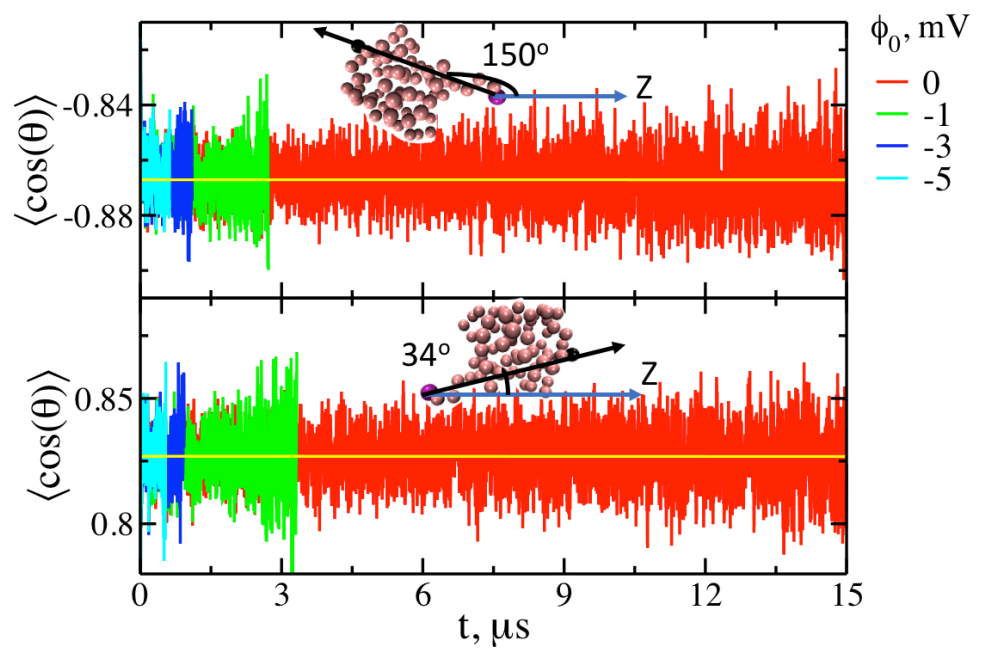

(b)

FIG. 6: (a) Average of z-component of the mean square displacement (z-MSD) as a function of time showing significant impact of $\phi_{0}$. For $\phi_{0}=0$, the z-MSD steadied around $100 \mathrm{~nm}^{2}$, whereas for other $\phi_{0}$, the z-MSD increased drastically as a function of time, showing that drift motion is induced even with slightest increase in surface potential. The z-MSD for positive $\phi_{0}$ are very close to the negative $\phi_{0}$ with the same magnitude (not shown). (b) The orientation of the principal axis of moment (vector from $76^{\text {th }}$ residue to $20^{\text {th }}$ residue) with respect to z-axis showed preferential orientation along nanopore due to elongated shape of Ubiquitin. The orientation angle is different based on the entry direction of the protein. It does not have much room to flip from its original orientation. 
small fluctuations in the protein structure can also be included to model a more realistic system without sacrificing too much of computational efficiency.

\section{ACKNOWLEDGEMENT}

Acknowledgement is made to the National Institutes of Health (Grant No. 5R01HG00277616), the National Science Foundation (DMR-2015935), and the AFOSR Grant FA9550-201-0142 for financial support. 


\section{REFERENCES}

${ }^{1}$ T. Ding, A. K. Chen, and Z. Lu, Science Bulletin 64, 1456 (2019).

${ }^{2}$ N. Varongchayakul, J. X. Song, A. Meller, and M. W. Grinstaff, Chemical Society Reviews 47 (2018), 10.1039/c8cs00106e.

${ }^{3}$ J. W. F. Robertson and J. E. Reiner, Proteomics 18 (2018), 10.1002/pmic.201800026.

${ }^{4}$ D. S. Talaga and J. L. Li, Journal of the American Chemical Society 131, 9287 (2009).

${ }^{5}$ G. Oukhaled, J. Mathe, A. L. Biance, L. Bacri, J. M. Betton, D. Lairez, J. Pelta, and

L. Auvray, Physical Review Letters 98 (2007), 10.1103/PhysRevLett.98.158101.

${ }^{6}$ D. Rodriguez-Larrea and H. Bayley, Nature Nanotechnology 8, 288 (2013).

${ }^{7}$ C. B. Rosen, D. Rodriguez-Larrea, and H. Bayley, Nature Biotechnology 32, 179 (2014).

${ }^{8}$ A. Oukhaled, B. Cressiot, L. Bacri, M. Pastoriza-Gallego, J. M. Betton, E. Bourhis, R. Jede, J. Gierak, L. Auvray, and J. Pelta, Acs Nano 5, 3628 (2011).

${ }^{9}$ R. Hu, J. J. Diao, J. Li, Z. P. Tang, X. Q. Li, J. Leitz, J. G. Long, J. K. Liu, D. P. Yu, and Q. Zhao, Scientific Reports 6 (2016), 10.1038/srep20776.

${ }^{10}$ R. J. Yu, S. M. Lu, S. W. Xu, Y. J. Li, Q. Xu, Y. L. Ying, and Y. T. Long, Chemical Science 10, 10728 (2019).

${ }^{11}$ X. Q. Li, X. Tong, W. L. Lu, D. P. Yu, J. J. Diao, and Q. Zhao, Nanoscale 11, 6480 (2019).

${ }^{12}$ T. Albrecht, Current Opinion in Electrochemistry 4, 159 (2017).

${ }^{13}$ T. Luchian, Y. Park, A. Asandei, I. Schiopu, L. Mereuta, and A. Apetrei, Accounts of Chemical Research 52, 267 (2019).

${ }^{14}$ A. Asandei, G. Di Muccio, I. Schiopu, L. Mereuta, I. S. Dragomir, M. Chinappi, and T. Luchian, Small Methods 4 (2020), 10.1002/smtd.201900595.

${ }^{15}$ R. Gao, Y. Lin, Y. L. Ying, and Y. T. Long, Science China-Chemistry 62, 1576 (2019).

${ }^{16}$ L. Restrepo-Perez, C. Joo, and C. Dekker, Nature Nanotechnology 13, 786 (2018).

${ }^{17}$ E. L. Bonome, F. Cecconi, and M. Chinappi, Nanoscale 11, 9920 (2019).

${ }^{18}$ F. Z. Hu, B. Angelov, S. Li, N. Li, X. B. Lin, and A. H. Zou, Chembiochem 21, 2467 (2020).

${ }^{19}$ D. E. Makarov, Accounts of Chemical Research 42, 281 (2009).

${ }^{20}$ D. Di Marino, E. L. Bonome, A. Tramontano, and M. Chinappi, Journal of Physical Chemistry Letters 6, 2963 (2015). 
${ }^{21}$ H. Chen, L. Li, T. Zhang, Z. W. Qiao, J. Tang, and J. Zhou, Journal of Physical Chemistry C 122, 2070 (2018).

${ }^{22}$ B. Lu, C. Stokes, M. Fahie, M. Chen, J. A. Golovchenko, and L. V. Hau, Biophysical Journal 115, 801 (2018).

${ }^{23}$ S. K. Kannam, S. C. Kim, P. R. Rogers, N. Gunn, J. Wagner, S. Harrer, and M. T. Downton, Nanotechnology 25 (2014), 10.1088/0957-4484/25/15/155502.

${ }^{24}$ N. Haridasan, S. K. Kannam, S. Mogurampelly, and S. P. Sathian, Physical Review E 97 (2018), 10.1103/PhysRevE.97.062415.

${ }^{25}$ J. Larkin, R. Y. Henley, M. Muthukumar, J. K. Rosenstein, and M. Wanunu, Biophysical Journal 106, 696 (2014).

${ }^{26}$ I. Nir, D. Huttner, and A. Meller, Biophys. J. 108, 2340 (2015).

${ }^{27}$ M. Muthukumar, J. Chem. Phys. 141, 081104 (2014).

${ }^{28}$ V. D. Ustach and R. Faller, European Physical Journal-Special Topics 225, 1643 (2016).

${ }^{29}$ Z. Y. Liu, X. R. Shi, and H. Y. Wu, Nanotechnology 30 (2019), 10.1088/1361-6528/aafdd7.

${ }^{30}$ S. Vijaykumar, C. E. Bugg, and W. J. Cook, J. Mol. Biol. 194, 531 (1987).

${ }^{31}$ S. H. Li and M. Hong, J. Am. Chem. Soc. 133, 1534 (2011).

${ }^{32}$ M. G. Fyta, S. Melchionna, E. Kaxiras, and S. Succi, Multiscale Modeling \& Simulation 5, 1156 (2006).

${ }^{33}$ C. T. A. Wong and M. Muthukumar, Journal of Chemical Physics 126 (2007), $10.1063 / 1.2723088$.

${ }^{34}$ J. C. Phillips, R. Braun, W. Wang, J. Gumbart, E. Tajkhorshid, E. Villa, C. Chipot, R. D. Skeel, L. Kale, and K. Schulten, J. Comput. Chem. 26, 1781 (2005).

${ }^{35}$ S. Plimpton, J. Comput. Phys. 117, 1 (1995).

${ }^{36}$ I. Kalcher and J. Dzubiella, Journal of Chemical Physics 130 (2009), 10.1063/1.3097530.

${ }^{37}$ T. F. Miller, M. Eleftheriou, P. Pattnaik, A. Ndirango, D. Newns, and G. J. Martyna, J. Chem. Phys. 116, 8649 (2002).

38.

${ }^{39}$ H. P. Erickson, Proc. Natl. Acad. Sci. U. S. A. 91, 10114 (1994).

${ }^{40}$ E. Walinda, D. Morimoto, M. Shirakawa, U. Scheler, and K. Sugase, Biochimica Et Biophysica Acta-General Subjects 1864 (2020), 10.1016/j.bbagen.2019.06.006.

${ }^{41}$ R. I. Stefureac, D. Trivedi, A. Marziali, and J. S. Lee, Journal of Physics-Condensed Matter 22 (2010), 10.1088/0953-8984/22/45/454133. 
bioRxiv preprint doi: https://doi.org/10.1101/2021.06.21.449278; this version posted June 22, 2021. The copyright holder for this preprint (which was not certified by peer review) is the author/funder. All rights reserved. No reuse allowed without permission.

${ }^{42}$ J. Sonnefeld, Colloids Surf., A 108, 27 (1996).

${ }^{43}$ H. Tian, W. Huang, M. Li, and M. Wang, Journal of Nanoscience and Nanotechnology 17, 6149 (2017). 